\section{S sciendo}

DOI: $10.2478 /$ awutm-2018-0015
Analele Universităţii de Vest,

Timişoara

Seria Matematică - Informatică

LVI, 2, (2018), 43- 70

\title{
Evolution of non-stationary processes and some maximum entropy principles
}

Vasile Preda and Irina Băncescu

Dedicated to Professor Mihail Megan on the occasion of his 70th birthday

\begin{abstract}
This paper studies, by using the speed-gradient principle, the evolution of non-stationary processes in the context of maximization of Varma, weighted Rényi, weighted Varma and Rényi-Tsallis of order $\alpha$ entropies.
\end{abstract}

AMS Subject Classification (2000). 60G35, 60F99, 60E99, 82B31, 82D99

Keywords. non-stationary processes, maximum entropy principle, Rényi entropy, Varma entropy, Rényi-Tsallis entropy, weighted entropy

\section{Introduction}

Entropies play an essential role in many fields such as physics, information theory, analysis of complex systems, electronics and engineering $[1,2,3]$. Among various entropies introduced in the specialized literature, we mention the Tsallis entropy $[4,6,5]$, Kaniadakis entropy [7, 8, 9], cumulative entropies [10], relative entropies [11, 12, 13], and weighted entropies $[14,15,16]$. Some well-known entropies are Shannon and Rényi entropies, the latter being a generalization of the former with both entropies having many applications, for example, the measurement of the electroencephalographic effects of desflurane and measures of heart rate $[17,18]$. Some applications of divergence measures involve Markov chains. Recently, $\alpha$-divergence and $\beta$-divergence 
for Markov chains have been generalized, weighted generalizations of these divergence measures being also considered [19, 20]. In 2018, Barbu et al. [21] have introduced and discussed generalized Cressie and Read power divergence class of measures for Markov chains. In 1966, a generalization with two parameters of the Rényi entropy was introduced, taking the name of Varma entropy. Rényi entropy has only one paramater, while Varma entropy has two paramaters, thus making it more flexible for different applications.

Let $\left(X_{t}\right)_{t \geq 0}$ be a non-stationary stochastic process defined over a probability space, where $X_{t}, t \geq 0$ is a continuous random variable. Suppose that $\Omega$ is the state space of the process and that $p(t, x)$ is the probability for which the process is in state $x$ at moment $t$. Also, we assume that $p(t, x)$ satisfies some continuity and/or differentiability hypothesis required with respect to $t$ for any $x \in \Omega$ and $t \geq 0$.

The Varma entropy of a random variable $X_{t}, t \geq 0$ is defined by the following equation $[22]$

$$
V\left(X_{t}, \alpha, \beta\right)=\frac{1}{\beta-\alpha} \log \int_{\Omega} p^{\alpha+\beta-1}(t, x) d x, \quad \beta \geq 1, \alpha \neq \beta, \beta-1<\alpha<\beta
$$

For $\beta=1$ and $\alpha \rightarrow 1$ Varma entropy reduces to Shannon entropy, while for $\beta=1$ we obtain the Rényi entropy. Recently, Varma entropy has been used to derive a generalized entropy of order statistics [23] and a generalized discrimination measure of order $(\alpha, \beta)$ [24].

Important principles related to the concept of entropy are the maximum and minimum entropy principles. Originated in statistical physics where they were applied in equilibrium problems for understanding the dynamics of systems, nowadays their applications have expanded to other areas of science and technology [25]. In econometrics, the maximum entropy principle states that given some constrains about the mean and Gini index, we can obtain probability densities that are least biased based on the prior information received, distributions which are derived by maximizing the entropy under the constrains given. This principle has been applied to obtain new Lorenz curve by maximizing the Tsallis entropy under equality and inequality constrains [26]. Considering the maximization of the Shannon entropy under mean and standard deviation constrains, the Gaussian distribution is obtained.

Recently, in 2016, Shalymov and Fradkov [27], applied the maximum entropy principle to analysis the dynamics of non-stationary processes that follow this principle. More, in 2016, the same problem was studied considering Tsallis statistics [28]. In [27], the authors proposed an evolution law of a system given by 


$$
\dot{p}=-\gamma(I-\Psi) p^{\beta-1},
$$

where $\dot{p}=\frac{d p}{d t}, I$ is an identity operator, $\Psi$ is a linear operator invariant to $p$, $\gamma>0$ is a constant gain and $\beta>0, \beta \neq 1$. Equation (1.4) has as solutions distributions that obey the maximum Rényi principle of parameter $\beta$.

In this paper, we study the evolution of non-stationary processes in the context of Varma, weighted Varma, weighted Rényi and Rényi-Tsallis of order $\alpha$ entropies maximization principles (the continuous case).

The proposed evolution law of a system in case of Varma entropy maximization is given by

$$
\dot{p}=-\gamma(I-\Psi) p^{\alpha+\beta-1},
$$

while for weighted Rényi entropy the proposed evolution law of a system is given by

$$
\dot{p}=-\gamma(I-\Psi) w p^{\beta-1},
$$

where $w$ is the weighted function and depends only on $x$. Here $\dot{p}$ is the derivative function of $p$ relative to $t$, continuous and integrable relative to $x$. Also, we suppose that hypotheses of derivability relative to $t$ and integrability relative to $x$ are satisfied throughout the paper and furthermore the expressions are finite.

The paper is organized as follows. Sections 2 and 3 describe the evolution of non-stationary systems that obey the maximum Varma and weighted Rényi entropy principles, respectively. Systems having a second constraint, namely total energy constraint are studied as well. Section 4 describes the evolution of non-stationary systems that obey the weighted Varma entropy principle. Section 5 describes the evolution of non-stationary systems that obey the Rényi-Tsallis entropy of order $\alpha$ principle while section 6 concludes the paper.

\section{Varma entropy and non-stationary systems}

Variational principles have long been applied in the study of dynamics of systems. Some variational principles are Gauss principle of least constraint, principle of minimum energy dissipation and Pontryagin maximum principle. The speed-gradient variational principle (SG) was first introduced in nonlinear control theory [29] having many applications. In 2008, Fradkov, formulated the SG principle for non-stationary processes [30] as follows. 
Consider a physical system described by systems of differential equations of the form

$$
\dot{x}=f(x, u, t)
$$

where $x=\left(x_{1}, x_{2}, \ldots, x_{n}\right)^{T}$ represents the systems states, $u=\left(u_{1}, u_{2}, \ldots, u_{m}\right)^{T}$ are the free (input) variables, and $\dot{x}=\frac{d x}{d t}, t \geq 0$. In order to model system dynamics, we search for the law of change of $u(t)$ subject to some criterion of behavior of the system given by the tendency to achieve a goal functional $W(x, t)$ set apriori (or more precisely the behavior is given by the decreasing of the goal functional).

Solution of the problem is obtained by deriving the speed of the goal functional $W(x, t)$, evaluate the gradient of the speed $\nabla_{u} \dot{W}$ with respect to input vector $u$ (speed-gradient vector), and formulate the law of dynamics as

$$
u=-\gamma \nabla_{u} \dot{W}(x, u, t) \text {, where } \gamma>0 \text { is a scalar. }
$$

An example of speed-gradient laws of dynamics is the motion of a particle in the potential field [30].

\subsection{Speed-gradient and maximum Varma entropy principles}

Based on the SG principle we derive the laws of motion for a non-stationary process which obeys the maximum Varma entropy principle. Similar to [27] we consider a system having a continuous distribution of possible states over a compact carrier, $\Omega$. The density function of the system, denoted by $p(t, x)$, satisfies the following condition

$$
\int_{\Omega} p(t, x) d x=1, \quad \forall t \geq 0
$$

and it is continuous over $\Omega$ except for a set having zero measure. From equation (2.3), we get the following restriction for the law of motion $u$

$$
\int_{\Omega} u(t, x) d x=0
$$

where $u(t, x)=\dot{p}(t, x)=\frac{d p}{d t}$.

Applying the SG principle for non-stationary processes as described above, we calculate the speed of the goal functional $\dot{\widetilde{V}}=\frac{d \widetilde{V}}{d t}$ as

$$
\dot{\tilde{V}}\left(X_{t}, \alpha, \beta\right)=-\left(\frac{\alpha+\beta-1}{\beta-\alpha}\right) \frac{\int_{\Omega} \dot{p}(t, x) p^{\alpha+\beta-2}(t, x) d x}{\int_{\Omega} p^{\alpha+\beta-1}(t, x) d x}
$$


where $\widetilde{V}=-V$.

The gradient of $\dot{\widetilde{V}}$ by $u$ is equal to

$$
\nabla_{u} \dot{\tilde{V}}\left(X_{t}, \alpha, \beta\right)=-\frac{\alpha+\beta-1}{\beta-\alpha} \frac{p^{\alpha+\beta-2}(t, x)}{\int_{\Omega} p^{\alpha+\beta-1}(t, x) d x} .
$$

According to the SG principle, we obtain the following evolution law

$$
u(t, x)=\gamma \frac{\alpha+\beta-1}{\beta-\alpha} \frac{p^{\alpha+\beta-2}(t, x)}{\int_{\Omega} p^{\alpha+\beta-1}(t, x) d x}+\lambda^{\prime} .
$$

where $\lambda^{\prime}=\lambda^{\prime}(t)$ is the Lagrange multiplier which obeys the following condition

$$
\int_{\Omega}\left(\gamma \frac{\alpha+\beta-1}{\beta-\alpha} \frac{p^{\alpha+\beta-2}(t, x)}{\int_{\Omega} p^{\alpha+\beta-1}(t, x) d x}+\lambda^{\prime}\right) d x=0
$$

From this we obtain the Lagrange multiplier as

$$
\lambda^{\prime}=-\left(\frac{(\alpha+\beta-1) \gamma}{(\beta-\alpha) \operatorname{mes}(\Omega)}\right) \frac{\int_{\Omega} p^{\alpha+\beta-2}(t, x) d x}{\int_{\Omega} p^{\alpha+\beta-1}(t, x) d x}
$$

where $\operatorname{mes}(\Omega)=\int_{\Omega} 1 d x$.

The system dynamics equation has the following form:

$$
\dot{p}(t, x)=\gamma \frac{\alpha+\beta-1}{\beta-\alpha} \frac{p^{\alpha+\beta-2}(t, x)}{\int_{\Omega} p^{\alpha+\beta-1}(t, x) d x}-\frac{(\alpha+\beta-1) \gamma}{(\beta-\alpha) \operatorname{mes}(\Omega)} \frac{\int_{\Omega} p^{\alpha+\beta-2}(t, x) d x}{\int_{\Omega} p^{\alpha+\beta-1}(t, x) d x}
$$

The general form of the evolution of the system is

$$
\dot{p}=-\frac{\gamma}{C_{t}}(\Psi-I) p^{\alpha+\beta-2}
$$

where $\Psi=\int_{\Omega}(\cdot) d x / \operatorname{mes}(\Omega)$ is a linear operator which is invariant to $p, I$ is an identity operator and $C_{t}=\frac{(\beta-\alpha) \int_{\Omega} p^{\alpha+\beta-1}(t, x) d x}{\alpha+\beta-1}$.

The stability of the obtained equilibrium equation (2.10) is derived considering the following Lyapunov function $M(p)=\widetilde{V}\left(X_{t}, \alpha, \beta\right)-\widetilde{V}_{\min } \geq 0$ where $\widetilde{V}_{\text {min }}$ is the minimum of $\widetilde{V}\left(X_{t}, \alpha, \beta\right)$ corresponding to equation (2.5). We have the following

$$
\dot{M}(p)=\dot{\widetilde{V}}\left(X_{t}, \alpha, \beta\right)=-\frac{\alpha+\beta-1}{\beta-\alpha} \frac{\int_{\Omega} u(t, x) p^{\alpha+\beta-2}(t, x) d x}{\int_{\Omega} p^{\alpha+\beta-1}(t, x) d x} .
$$

We substitute the expression for $u$ from (2.10) and obtain 


$$
\begin{aligned}
\dot{M}(p) & =\frac{\gamma(\alpha+\beta-1)^{2}}{\operatorname{mes}(\Omega)(\beta-\alpha)^{2}\left(\int_{\Omega} p^{\alpha+\beta-1}(t, x) d x\right)^{2}}\left[\left(\int_{\Omega} p^{\alpha+\beta-2}(t, x) d x\right)^{2}\right. \\
& \left.-\operatorname{mes}(\Omega) \int_{\Omega}\left(p^{\alpha+\beta-2}(t, x)\right)^{2} d x\right]
\end{aligned}
$$

Applying the Cauchy Bunyakovsky Schwarz (CBS) inequality

$$
\left|\int_{\Omega} f(x) g(x) d x\right|^{2} \leq\left(\int_{\Omega}|f(x)|^{2} d x\right)\left(\int_{\Omega}|g(x)|^{2} d x\right)
$$

for $f=p^{\alpha+\beta-2}$ and $g=1$, we obtain $\dot{M}(p) \leq 0(\gamma>0)$. We have that $\dot{M}(p)=0$ is true when $p^{\alpha+\beta-2}(t, x)=\sigma_{t}=$ constant for all $t \geq 0$ which is possible only if we take $p(t, x)=C=$ const. Applying equation (2.3), we get $C_{t}=\frac{(\beta-\alpha) \sigma_{t}}{\alpha+\beta-1}$ and prove that $\sigma_{t}$ does not depend on $t$, meaning $C_{t}$ also does not depend on $t$. From the same equation (2.3), we derive constant $C$ to be $C=(\operatorname{mes}(\Omega))^{-1}$. Therefore, there is only one unique density function $p^{*}=(\operatorname{mes}(\Omega))^{-1}$ for the equilibrium state of the system which evolves by evolution law given by (2.10).

\section{Asymptotic convergence}

Asymptotic convergence of all solutions to $p^{*}$ can be proved by applying Barbalat's lemma [37].

Lemma 2.1 (Barbalat's lemma). If differentiable function $f(t)$ has a finite limit for $t \rightarrow \infty$ and its derivative $\dot{f}(t)$ is uniformly continuous then $\dot{f}(t) \rightarrow 0$ for $t \rightarrow 0$.

Theorem 2.1. If $p(t, x)$ is a density function defined by equation (2.10), then for any $x$ we have $\lim _{t \rightarrow \infty} p(t, x)=p^{*}(x)$.

Proof. Similar to [27], the theorem can be proven by calculating $\ddot{M}(p)$ and applying Barbalat's lemma.

We have

$$
\begin{aligned}
\ddot{M}(p) & =\frac{\alpha+\beta-1}{\beta-\alpha} \frac{\int_{\Omega}\left(\dot{u}(t, x) p^{\alpha+\beta-2}(t, x)+(\alpha+\beta-2) u^{2}(t, x) p^{\alpha+\beta-3}(t, x)\right) d x}{\int_{\Omega} p^{\alpha+\beta-1}(t, x) d x} \\
& -\frac{(\alpha+\beta-1)^{2}}{\beta-\alpha} \int_{\Omega} u(t, x) p^{\alpha+\beta-2}(t, x) d x \frac{\int_{\Omega} p^{\alpha+\beta-2}(t, x) u(t, x) d x}{\left(\int_{\Omega} p^{\alpha+\beta-1}(t, x) d x\right)^{2}} .
\end{aligned}
$$


It can be shown that function $|\ddot{M}(p)|$ is bounded over compact carrier $\Omega$, hence $\dot{M}(p)$ is uniformly continuous. Applying Barbalat's lemma, we have $\dot{M}(p) \rightarrow 0$ for $t \rightarrow 0$. Rewriting $\dot{M}(p)$ as

$$
\dot{M}(p)=-\frac{\gamma(\alpha+\beta-1)^{2}}{m e s(\Omega)(\beta-\alpha)^{2}} \frac{\left\|p^{\alpha+\beta-2}\right\|^{2}}{\left(p^{\alpha+\beta-1}, 1\right)^{2}}\left(1-\cos ^{2}(\gamma(t))\right)
$$

where $\gamma(t)=\cos \left(\left(1, p^{\alpha+\beta-2}\right)\right),(1, f)=\int_{\Omega} f(x) d x$ and $\|f\|^{2}=\int_{\Omega} f^{2}(x) d x$.

From constraint $(2.3)$ we obtain $\gamma(t) \rightarrow 0$, meaning $\bar{p}^{\alpha+\beta-2}(t, x) \rightarrow \overline{1}$, where $\bar{p}^{\alpha+\beta-2}(t, x)$ and $\overline{1}$ are normalized values for $p^{\alpha+\beta-2}(t, x)$ and 1 , respectively. Hence, we have $p(t, x) \rightarrow p^{*}$ for $t \rightarrow \infty$, and for all $p(t, x)$ that follow the evolution law (2.10).

\subsection{Total energy constraint}

Consider a system having a total energy conservation constraint given by [27]

$$
\int_{\Omega} p(t, x) h(x) d x=E
$$

where $E$ is the common energy of a system and $h$ is the density of energy, $h \in L_{2}(\Omega), h$ is not a constant function. The dynamics equation of this system is defined as [27]

$$
u=-\gamma \nabla_{u} \dot{V}+\lambda_{1} h+\lambda_{2}
$$

The constraints (2.3) and (2.16) are equivalent to

$$
\int_{\Omega} u(t, x) d x=0 \quad \text { and } \quad \int_{\Omega} u(t, x) h(x) d x=0, \quad \forall t \geq 0, \quad \text { respectively }
$$

Based on the above constraints, the Lagrange multipliers $\lambda_{1}$ and $\lambda_{2}$ are

$$
\begin{aligned}
\lambda_{1} & =\frac{\gamma(\alpha+\beta-1)}{(\beta-\alpha)\left(\int_{\Omega} p^{\alpha+\beta-1}(t, x) d x\right)} \\
& \times \frac{\operatorname{mes}(\Omega) \int_{\Omega} p^{\alpha+\beta-2}(t, x) h(x) d x-\left(\int_{\Omega} p^{\alpha+\beta-2}(t, x) d x\right)\left(\int_{\Omega} h(x) d x\right)}{\operatorname{mes}(\Omega) \int_{\Omega} h^{2}(x) d x-\left(\int_{\Omega} h(x) d x\right)^{2}}
\end{aligned}
$$




$$
\begin{aligned}
\lambda_{2} & =\frac{\gamma(\alpha+\beta-1)}{(\beta-\alpha)\left(\int_{\Omega} p^{\alpha+\beta-1}(t, x) d x\right)} \\
& \times \frac{\left(\int_{\Omega} p^{\alpha+\beta-2}(t, x) d x\right)\left(\int_{\Omega} h^{2}(x) d x\right)-\left(\int_{\Omega} p^{\alpha+\beta-2}(t, x) h(x) d x\right)\left(\int_{\Omega} h(x) d x\right)}{\operatorname{mes}(\Omega) \int_{\Omega} h^{2}(x) d x-\left(\int_{\Omega} h(x) d x\right)^{2}}
\end{aligned}
$$

Since $h$ is not a constant function, the quantity mes $(\Omega) \int_{\Omega} h^{2}(x) d x-\left(\int_{\Omega} h(x) d x\right)^{2}$ is different from zero.

\section{Equilibrium stability}

Using the same Lyapunov function $M$ used in the previous section, for $\widetilde{V}$ and Lagrange multipliers $\lambda_{1}^{\prime}=-\lambda_{1}$ and $\lambda_{2}^{\prime}=-\lambda_{2}$, we obtain the following expression

$$
\begin{aligned}
\dot{M}(p) & =\frac{\gamma(\alpha+\beta-1)^{2}}{\operatorname{mes}(\Omega)(\beta-\alpha)^{2}\left(\int_{\Omega} p^{\alpha+\beta-1}(t, x) d x\right.} \\
& \times \frac{\left[\operatorname{mes}(\Omega) \int_{\Omega} p^{\alpha+\beta-2}(t, x) h(x) d x-\int_{\Omega} h(x) d x \int_{\Omega} p^{\alpha+\beta-2}(t, x) d x\right]^{2}}{\operatorname{mes}(\Omega) \int_{\Omega} h^{2}(x) d x-\left(\int_{\Omega} h(x) d x\right)^{2}} \\
& -\frac{\gamma(\alpha+\beta-1)^{2}}{\operatorname{mes}(\Omega)(\beta-\alpha)^{2}\left(\int_{\Omega} p^{\alpha+\beta-1}(t, x) d x\right)^{2}} \\
& \times\left[\operatorname{mes}(\Omega) \int_{\Omega}\left(p^{\alpha+\beta-2}(t, x)\right)^{2} d x-\left(\int_{\Omega} p^{\alpha+\beta-2}(t, x) d x\right)^{2}\right] .
\end{aligned}
$$

Applying inequality

$$
<f, g>^{2} \leq<f, f><g, g>
$$

from [27] where functional $<,>: L_{2}(\Omega) \times L_{2}(\Omega) \rightarrow \mathbb{R}, f, g \in L_{2}(\Omega)$ is defined as

$$
<f, g>=\operatorname{mes}(\Omega) \int_{\Omega} f g d x-\int_{\Omega} f d x \int_{\Omega} g d x .
$$

for $f=p^{\alpha+\beta-2}$ and $g=h$, we get 


$$
\begin{aligned}
& \left(\operatorname{mes}(\Omega) \int_{\Omega} h(x) p^{\alpha+\beta-2}(t, x) d x-\int_{\Omega} h(x) d x \int_{\Omega} p^{\alpha+\beta-2}(t, x) d x\right)^{2} \\
& \leq\left(\operatorname{mes}(\Omega) \int_{\Omega}\left(p^{\alpha+\beta-2}(t, x)\right)^{2} d x-\left(\int_{\Omega} p^{\alpha+\beta-2}(t, x) d x\right)^{2}\right) \\
& \quad \times\left(\operatorname{mes}(\Omega) \int_{\Omega} h^{2}(x) d x-\left(\int_{\Omega} h(x) d x\right)^{2}\right)
\end{aligned}
$$

Hence, we obtain $\dot{M}(p) \leq 0 . \dot{M}(p)=0$ holds if and only if

$$
\exists \sigma_{t}, \rho_{t} \in \mathbb{R} \quad \text { such that } \quad p^{\alpha+\beta-2}=\sigma_{t} h+\rho_{t}, \quad \forall t \geq 0,
$$

The density equilibrium is given by

$$
p^{\alpha+\beta-2}(t, x)=\frac{C_{t} \lambda_{1}^{\prime} h(x)}{\gamma}+\frac{C_{t} \lambda_{2}^{\prime}}{\gamma}
$$

where $C_{t}=(\beta-\alpha) \int_{\Omega} p^{\alpha+\beta-1}(t, x) d x /(\alpha+\beta-2), \alpha+\beta>2$. If $\alpha+\beta=3$, applying equations $(2.3),(2.16)$ and $(2.23)$, we have that $\sigma_{t}=\sigma=\lambda_{1}^{\prime}\left(C_{t} / \gamma\right)$ and $\rho_{t}=\rho=\lambda_{2}^{\prime}\left(C_{t} / \gamma\right)$ are independent of $t$. In this case, we have an unique distribution for the equilibrium state $p^{*}(x)$.

\section{Asymptotic convergence}

Asymptotic convergence is proven in this case in a similar manner as in the previous section by applying Barbalat's lemma.

Theorem 2.2. If $p(t, x)$ is a density function defined by $u=-\gamma \nabla_{u} \dot{\hat{H}^{w}}+$ $\lambda_{1}^{\prime} h+\lambda_{2}^{\prime}$, then for any $x$ we have $\lim _{t \rightarrow \infty} p(t, x)=p^{*}(x)$.

\section{Weighted Rényi entropy and non-stationary systems}

Rényi entropy is a generalization of Shannon entropy being a monotonic function of the information and a scale dependent function in the continuous case [31, 32]. Rényi entropy is used in classical string-net models, classification of daily electrical load patterns, signal segmentation and classification and identification of frog sound $[33,34,35,36]$. 
The Rényi entropy of a random variable $X_{t}, t \geq 0$ is defined as

$$
H\left(X_{t}, \beta\right)=\frac{1}{1-\beta} \log \int_{\Omega} p^{\beta}(t, x) d x, \quad \beta>0, \beta \neq 1 .
$$

Weighted entropies were introduced in 1968 by Belis and Guiasu [14] and in 1971 by Guiasu [15]. The main concept around weighted entropy is that it is designed to take the importance of data/states under consideration. Therefore, weights are given to every state of a system according to their importance.

The weighted Rényi entropy of a random variable $X_{t}, t \geq 0$ is defined as

$$
H^{w}\left(X_{t}, \beta\right)=\frac{1}{1-\beta} \log \int_{\Omega} w(x) p^{\beta}(t, x) d x, \quad \beta>0, \beta \neq 1 .
$$

where $w$ is a positive weighted function, $w \in L_{2}(\Omega)$. An example of a weighted function is $w(x)=e^{x}$. For $w(x)=1$ for all $x$, we obtain the Rényi entropy.

\subsection{Speed-gradient and maximum weighted Rényi entropy prin- ciples}

Applying the SG principle for non-stationary processes, we calculate the speed of the goal functional $\frac{\dot{H^{w}}}{d t}=\frac{d \widetilde{H^{w}}}{d t}$ as

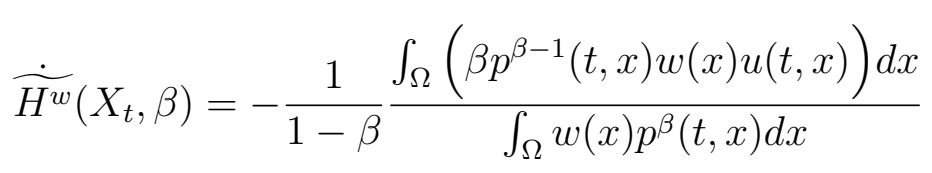

where $\widetilde{H^{w}}=-H^{w}$.

The gradient of $\dot{\overrightarrow{H^{w}}}$ by $u$ is equal to

$$
\nabla_{u} \dot{\widetilde{H^{w}}}\left(X_{t}, \beta\right)=-\frac{\beta}{1-\beta} \frac{p^{\beta-1}(t, w) w(x)}{\int_{\Omega} w(x) p^{\beta}(t, x) d x}
$$

According to the SG principle, we obtain the following evolution law

$$
u=\frac{\beta \gamma}{1-\beta} \frac{w(x) p^{\beta-1}(t, x)}{\int_{\Omega} w(x) p^{\beta}(t, x) d x}+\lambda^{\prime}
$$

where $\lambda^{\prime}$ is the Lagrange multiplier which obeys the following condition

$$
\int_{\Omega}\left(\frac{\beta \gamma}{1-\beta} \frac{w(x) p^{\beta-1}(t, x)}{\int_{\Omega} w(x) p^{\beta}(t, x) d x}+\lambda^{\prime}\right) d x=0
$$


From this we obtain the Lagrange multiplier as

$$
\lambda^{\prime}=-\frac{\beta \gamma}{(1-\beta) \operatorname{mes}(\Omega)} \frac{\int_{\Omega} w(x) p^{\beta-1}(t, x) d x}{\int_{\Omega} w(x) p^{\beta}(t, x) d x}
$$

The system dynamics equation has the following form:

$$
\dot{p}(t, x)=\frac{\beta \gamma}{1-\beta} \frac{w(x) p^{\beta-1}(t, x)}{\int_{\Omega} w(x) p^{\beta}(t, x) d x}-\frac{\beta \gamma}{(1-\beta) \operatorname{mes}(\Omega)} \frac{\int_{\Omega} w(x) p^{\beta-1}(t, x) d x}{\int_{\Omega} w(x) p^{\beta}(t, x) d x}
$$

The general form of the evolution of the system is

$$
\dot{p}=-\frac{\gamma}{C_{t}}(\Psi-I) w p^{\beta-1}
$$

where $\Psi=\int_{\Omega}(\cdot) d x / \operatorname{mes}(\Omega)$ is a linear operator which is invariant to $p, I$ is an identity operator and $C_{t}=(1-\beta) \int_{\Omega} w(x) p^{\beta}(t, x) / \beta$.

The stability of the obtained equilibrium equation (3.8) is derived considering the following Lyapunov function $M(p)=\widetilde{H^{w}}\left(X_{t}, \alpha, \beta\right)-{\widetilde{H^{w}}}_{\text {min }} \geq 0$ where $\widetilde{H^{w}}{ }_{\text {min }}$ is the minimum of $\widetilde{H^{w}}\left(X_{t}, \alpha, \beta\right)$ corresponding to equation (2.3). We have the following

$$
\dot{M}(p)=\dot{\widetilde{H^{w}}}(X, \beta)=-\frac{1}{1-\beta} \frac{\int_{\Omega}\left(\beta p^{\beta-1}(t, x) w(x) u(t, x)\right) d x}{\int_{\Omega} w(x) p^{\beta}(t, x) d x}
$$

We substitute the expression for $u$ from (3.8) and obtain

$$
\begin{aligned}
\dot{M}(p) & =\frac{\gamma \beta^{2}}{m e s(\Omega)(1-\beta)^{2}\left(\int_{\Omega} w(x) p^{\beta}(t, x) d x\right)^{2}}\left\{\left(\int_{\Omega} w(x) p^{\beta-1}(t, x) d x\right)^{2}\right. \\
& \left.-\operatorname{mes}(\Omega) \int_{\Omega}\left(w(x) p^{\beta-1}(t, x)\right)^{2} d x\right\}
\end{aligned}
$$

Applying the CBS inequality for $f=w p^{\beta-1}$ and $g=1$, since $\gamma>0$ we obtain $\dot{M}(p) \leq 0 . \dot{M}(p)=0$ holds only if $w p^{\beta-1}=\sigma_{t}=$ constant meaning when $p(t, x)=\left(\frac{C}{w(x)}\right)^{1 /(\beta-1)}$. Applying equation (2.3), we prove that $\sigma_{t}$ does not depend on $t$. Therefore, there is only one unique density function corresponding to the maximum entropy distribution of (2.3) for the equilibrium state of the system which has as evolution law given by equation (3.8). 


\section{Asymptotic convergence}

Asymptotic convergence in this case can be obtained similar to the previous case applying Barbalat's lemma and by considering that the weighted function $w$ is bounded.

Theorem 3.1. If $p(t, x)$ is a density function defined by equation (3.8), then for any $x$ we get $\lim _{t \rightarrow \infty} p(t, x)=p^{*}(x)$.

\subsection{Total energy constraint}

Consider a system having a total energy conservation constraint given by [27]

$$
\int_{\Omega} p(t, x) h(x) d x=E,
$$

where $E$ is the common energy of a system and $h$ is the density of energy, $h \in L_{2}(\Omega), h$ is not a constant function. The dynamics equation of this system is defined as [27]

$$
u=-\gamma \nabla_{u} \dot{H}^{w}+\lambda_{1} h+\lambda_{2} .
$$

Considering constraints (2.3) and (3.11), the Lagrange multipliers $\lambda_{1}$ and $\lambda_{2}$ are

$$
\begin{aligned}
\lambda_{1} & =\frac{\beta \gamma}{(1-\beta)\left(\int_{\Omega} p^{\beta}(t, x) w(x) d x\right)} \\
& \times \frac{\operatorname{mes}(\Omega) \int_{\Omega} p^{\beta-1}(t, x) w(x) h(x) d x-\left(\int_{\Omega} p^{\beta-1}(t, x) w(x) d x\right)\left(\int_{\Omega} h(x) d x\right)}{\operatorname{mes}(\Omega) \int_{\Omega} h^{2}(x) d x-\left(\int_{\Omega} h(x) d x\right)^{2}} \\
\lambda_{2} & =\frac{\beta \gamma}{(1-\beta)\left(\int_{\Omega} p^{\beta}(t, x) w(x) d x\right)} \frac{1}{m e s(\Omega) \int_{\Omega} h^{2}(x) d x-\left(\int_{\Omega} h(x) d x\right)^{2}} \\
& \times\left\{\left(\int_{\Omega} p^{\beta-1}(t, x) w(x) d x\right)\left(\int_{\Omega} h^{2}(x) d x\right)-\left(\int_{\Omega} p^{\beta-1}(t, x) w(x) h(x) d x\right)\right. \\
& \left.\times\left(\int_{\Omega} h(x) d x\right)\right\}
\end{aligned}
$$




\section{Equilibrium stability}

Using the same Lyapunov function $M$ used in the previous section, where ${\widetilde{H^{w}}}_{\text {min }}$ is the minimum of $\widetilde{H^{w}}$ corresponding to (2.3) and (3.11), for $\widetilde{H^{w}}$ and Lagrange multipliers $\lambda_{1}^{\prime}=-\lambda_{1}$ and $\lambda_{2}^{\prime}=-\lambda_{2}$, we obtain the following expression

$$
\begin{aligned}
& \dot{M}(p)=\frac{\gamma \beta^{2}}{m e s(\Omega)(1-\beta)^{2}\left(\int_{\Omega} p^{\beta}(t, x) w(x) d x\right)^{2}} \\
& \times \frac{\left[\operatorname{mes}(\Omega) \int_{\Omega} p^{\beta-1}(t, x) w(x) h(x) d x-\int_{\Omega} h(x) d x \int_{\Omega} p^{\beta-1}(t, x) w(x) d x\right]^{2}}{m e s(\Omega) \int_{\Omega} h^{2}(x) d x-\left(\int_{\Omega} h(x) d x\right)^{2}} \\
& -\frac{\gamma \beta^{2}}{\operatorname{mes}(\Omega)(1-\beta)^{2}\left(\int_{\Omega} p^{\beta}(t, x) w(x) d x\right)^{2}}\left[\operatorname{mes}(\Omega) \int_{\Omega}\left(p^{\beta-1}(t, x) w(x)\right)^{2} d x\right. \\
& \left.-\left(\int_{\Omega} p^{\beta-1}(t, x) w(x) d x\right)^{2}\right]
\end{aligned}
$$

Applying inequality (2.22) for $f=p^{\beta-1} w$ and $g=h$, we get

$$
\begin{aligned}
& \left(\operatorname{mes}(\Omega) \int_{\Omega} h(x) w(x) p^{\beta-1}(t, x) d x-\int_{\Omega} h(x) d x \int_{\Omega} p^{\beta-1}(t, x) w(x) d x\right)^{2} \\
& \leq\left(\operatorname{mes}(\Omega) \int_{\Omega}\left(p^{\beta-1}(t, x) w(x)\right)^{2} d x-\left(\int_{\Omega} p^{\beta-1}(t, x) w(x) d x\right)^{2}\right) \\
& \quad \times\left(\operatorname{mes}(\Omega) \int_{\Omega} h^{2}(x) d x-\left(\int_{\Omega} h(x) d x\right)^{2}\right)
\end{aligned}
$$

Hence, we obtain $\dot{M}(p) \leq 0 . \dot{M}(p)=0$ holds if and only if

$$
\exists \quad \sigma_{t}, \rho_{t} \in \mathbb{R} \text { such that } w p^{\beta-1}=\sigma_{t} h+\rho_{t} .
$$

Thus,

$$
p^{\beta-1}(t, x)=\sigma_{t} \frac{h(x)}{w(x)}+\frac{\rho_{t}}{w(x)}, \quad \forall x \in \Omega
$$

The general form of the equilibrium density is given by

$$
w(x) p^{\beta-1}(t, x)=\frac{C_{t} \lambda_{1}^{\prime} h(x)}{\gamma}+\frac{C_{t} \lambda_{2}^{\prime}}{\gamma}
$$


where $C_{t}=(\beta-1) \int_{\Omega} p^{\beta}(t, x) w(x) d x / \beta$. If $\beta=2$, applying equations (2.3), (3.11) and (3.16), we have that $C_{t}$ does not depend on $t$, and therefore $\sigma_{t}$ and $\rho_{t}$ do not depend on $t$. Hence, we get $\sigma_{t}=\sigma=\lambda_{1}^{\prime}\left(C_{t} / \gamma\right)$ and $\rho_{t}=\rho=\lambda_{2}\left(C_{t} / \gamma\right)$, having an unique density function $p^{*}(x)$ for an equilibrium state distribution corresponding to the maximum entropy distribution of equations (2.3) and (3.11) of a non-stationary system which obeys evolution equation (3.12).

\section{Asymptotic convergence}

Asymptotic convergence in this case can be obtained similar to the previous case applying Barbalat's lemma and by considering that the weighted function $w$ is bounded.

Theorem 3.2. For all density functions defined by equation $u=-\gamma \nabla_{u} \dot{H^{w}}+$ $\lambda_{1}^{\prime} h+\lambda_{2}^{\prime}$, it is true that $p(t, x) \rightarrow p^{*}(x)$ for $t \rightarrow \infty$ and for any $x \in \Omega$.

\section{Weighted Varma entropy and non-stationary sys- tems}

In this section, we introduce the weighted Varma entropy of a random variable $X_{t}$ defined as

$$
V^{w}\left(X_{t}, \alpha, \beta\right)=\frac{1}{\beta-\alpha} \log \int_{\Omega} w(x) p^{\alpha+\beta-1}(t, x) d x,
$$

where $\beta \geq 1, \alpha \neq \beta, \beta-1<\alpha<\beta$, and $w$ is a positive weighted function.

\subsection{Speed-gradient and maximum weighted Varma entropy prin- ciples}

Applying the SG principle for non-stationary processes, we calculate the speed of the goal functional $\dot{V^{w}}=\frac{d \widetilde{V^{w}}}{d t}$ as

$$
\dot{\widetilde{V^{w}}}\left(X_{t}, \alpha, \beta\right)=-\frac{1}{\beta-\alpha} \frac{\int_{\Omega}\left((\alpha+\beta-1) p^{\alpha+\beta-2}(t, x) w(x) u(t, x)\right) d x}{\int_{\Omega} w(x) p^{\alpha+\beta-1}(t, x) d x}
$$

where $\widetilde{V^{w}}=-V^{w}$ 
The gradient of $\dot{V^{w}}$ by $u$ is equal to

$$
\nabla_{u} \dot{\widetilde{V^{w}}}\left(X_{t}, \alpha, \beta\right)=-\frac{\alpha+\beta-1}{\beta-\alpha} \frac{p^{\alpha+\beta-2}(t, x) w(x)}{\int_{\Omega} w(x) p^{\alpha+\beta-1}(t, x) d x} .
$$

According to the SG principle, we obtain the following evolution law

$$
u=\frac{(\alpha+\beta-1) \gamma}{\beta-\alpha} \frac{p^{\alpha+\beta-2}(t, x) w(x)}{\int_{\Omega} w(x) p^{\alpha+\beta-1}(t, x) d x}+\lambda^{\prime},
$$

where $\lambda^{\prime}$ is the Lagrange multiplier which obeys the following condition

$$
\int_{\Omega}\left(\frac{(\alpha+\beta+1) \gamma}{\beta-\alpha} \frac{p^{\alpha+\beta-2}(t, x) w(x)}{\int_{\Omega} w(x) p^{\alpha+\beta-1}(t, x) d x}+\lambda^{\prime}\right) d x=0
$$

From this we obtain the Lagrange multiplier as

$$
\lambda^{\prime}=-\frac{(\alpha+\beta-1) \gamma}{(\beta-\alpha) \operatorname{mes}(\Omega)} \frac{\int_{\Omega} w(x) p^{\alpha+\beta-2}(t, x) d x}{\int_{\Omega} w(x) p^{\alpha+\beta-1}(t, x) d x}
$$

The system dynamics equation has the following form:

$$
\begin{aligned}
\dot{p}(t, x) & =\frac{(\alpha+\beta-1) \gamma}{\beta-\alpha} \frac{p^{\alpha+\beta-2}(t, x) w(x)}{\int_{\Omega} w(x) p^{\alpha+\beta-1}(t, x) d x} \\
& -\frac{(\alpha+\beta-1) \gamma}{(\beta-\alpha) \operatorname{mes}(\Omega)} \frac{\int_{\Omega} w(x) p^{\alpha+\beta-2}(t, x) d x}{\int_{\Omega} w(x) p^{\alpha+\beta-1}(t, x) d x}
\end{aligned}
$$

The general form of the evolution of the system is

$$
\dot{p}=-\frac{\gamma}{C_{t}}(\Psi-I) w p^{\alpha+\beta-2}
$$

where $\Psi=\int_{\Omega}(\cdot) d x / \operatorname{mes}(\Omega)$ is a linear operator which is invariant to $p, I$ is an identity operator and $C_{t}=(\beta-\alpha) \int_{\Omega} w(x) p^{\alpha+\beta-1}(t, x) /(\alpha+\beta-1)$.

The stability of the obtained equilibrium equation (4.7) is derived considering the following Lyapunov function $M(p)=\widetilde{V^{w}}\left(X_{t}, \alpha, \beta\right)-{\widetilde{V^{w}}}_{\text {min }} \geq 0$ where ${\widetilde{V^{w}}}_{\text {min }}$ is the minimum of $\widetilde{V^{w}}\left(X_{t}, \alpha, \beta\right)$ corresponding to $(2.3)$.

We have the following

$$
\dot{M}(p)=\dot{\widetilde{V^{w}}}\left(X_{t}, \alpha, \beta\right)=-\frac{1}{\beta-\alpha} \frac{\int_{\Omega}\left((\alpha+\beta-1) p^{\alpha+\beta-2}(t, x) w(x) u(t, x)\right) d x}{\int_{\Omega} w(x) p^{\alpha+\beta-1}(t, x) d x}
$$


We substitute the expression for $u$ from (4.7) and obtain

$$
\begin{aligned}
\dot{M}(p) & =\frac{\gamma(\alpha+\beta-1)^{2}}{\operatorname{mes}(\Omega)(\beta-\alpha)^{2}\left(\int_{\Omega} w(x) p^{\alpha+\beta-1}(t, x) d x\right)^{2}} \\
& \times\left\{\left(\int_{\Omega} w(x) p^{\alpha+\beta-1}(t, x) d x\right)^{2}-\operatorname{mes}(\Omega) \int_{\Omega}\left(w(x) p^{\alpha+\beta-1}(t, x)\right)^{2} d x\right\}
\end{aligned}
$$

Applying the CBS inequality for $f=w p^{\alpha+\beta-1}$ and $g=1$, we obtain $\dot{M}(p) \leq 0$ $(\gamma>0) . \dot{M}(p)=0$ holds only if $w p^{\alpha+\beta-2}=\sigma_{t}=$ constant, meaning when $p(t, x)=C=p^{*}(x)$. Applying equation (2.3), we prove that $\sigma_{t}$, and therefore $C_{t}$, does not depend on $t$. From constraint (2.3), we get $C=\left(\frac{\sigma}{w(x)}\right)^{1 /(\alpha+\beta-2)}$. Therefore, there is only one unique density function $p^{*}(x)=\left(\frac{\sigma}{w(x)}\right)^{1 /(\alpha+\beta-2)}$ corresponding to the maximum entropy distribution of (2.3) for the equilibrium state of the system which has as evolution law given by equation (4.7).

\section{Asymptotic convergence}

Asymptotic convergence in this case can be obtained similar to the previous case applying Barbalat's lemma and by considering that the weighted function $w$ is bounded.

Theorem 4.1. If $p(t, x)$ is a density function defined by equation (4.7), then for any $x$ we get $\lim _{t \rightarrow \infty} p(t, x)=p^{*}(x)$.

\subsection{Total energy constraint}

Consider a system having a total energy conservation constraint given by [27]

$$
\int_{\Omega} p(t, x) h(x) d x=E,
$$

where $E$ is the common energy of a system and $h$ is the density of energy, $h \in L_{2}(\Omega), h$ is not a constant function.

The dynamics equation of this system is defined as [27]

$$
u=-\gamma \nabla_{u} \dot{V^{w}}+\lambda_{1} h+\lambda_{2} .
$$

Considering constraints (2.3) and (4.9), the Lagrange multipliers $\lambda_{1}$ and $\lambda_{2}$ are 


$$
\begin{aligned}
\lambda_{1} & =\frac{(\alpha+\beta-1) \gamma}{(\beta-\alpha)\left(\int_{\Omega} p^{\alpha+\beta-1}(t, x) w(x) d x\right)} \\
\times & \frac{\operatorname{mes}(\Omega) \int_{\Omega} p^{\alpha+\beta-2}(t, x) w(x) h(x) d x-\left(\int_{\Omega} p^{\alpha+\beta-2}(t, x) w(x) d x\right)\left(\int_{\Omega} h(x) d x\right)}{\operatorname{mes}(\Omega) \int_{\Omega} h^{2}(x) d x-\left(\int_{\Omega} h(x) d x\right)^{2}} \\
\lambda_{2} & =\frac{(\alpha+\beta-1) \gamma}{(\beta-\alpha)\left(\int_{\Omega} p^{\alpha+\beta-1}(t, x) w(x) d x\right)} \frac{1}{\operatorname{mes}(\Omega) \int_{\Omega} h^{2}(x) d x-\left(\int_{\Omega} h(x) d x\right)^{2}} \\
& \times\left\{\left(\int_{\Omega} p^{\alpha+\beta-2}(t, x) w(x) d x\right)\left(\int_{\Omega} h^{2}(x) d x\right)\right. \\
& \left.-\left(\int_{\Omega} p^{\alpha+\beta-2}(t, x) w(x) h(x)\right)\left(\int_{\Omega} h(x) d x\right)\right\}
\end{aligned}
$$

\section{Equilibrium stability}

Using the same Lyapunov function $M(p)$ used in the previous section, where ${\widetilde{V^{w}}}_{\min }$ is the minimum of $\widetilde{V^{w}}$ satisfying equation (2.3) and (4.9), for $\widetilde{V^{w}}$ and Lagrange multipliers $\lambda_{1}^{\prime}=-\lambda_{1}$ and $\lambda_{2}^{\prime}=-\lambda_{2}$, we obtain the following expression

$$
\begin{aligned}
& \dot{M}(p)=\frac{\gamma(\alpha+\beta-1)^{2}}{\operatorname{mes}(\Omega)(\beta-\alpha)^{2}\left(\int_{\Omega} p^{\alpha+\beta-1}(t, x) w(x) d x\right)^{2}} \\
& \times \frac{\left[\operatorname{mes}(\Omega) \int_{\Omega} p^{\alpha+\beta-2}(t, x) w(x) h(x) d x-\int_{\Omega} h(x) d x \int_{\Omega} p^{\alpha+\beta-2}(t, x) w(x) d x\right]^{2}}{\operatorname{mes}(\Omega) \int_{\Omega} h^{2}(x) d x-\left(\int_{\Omega} h(x) d x\right)^{2}} \\
& -\frac{\gamma(\alpha+\beta-1)^{2}}{\operatorname{mes}(\Omega)(\beta-\alpha)^{2}\left(\int_{\Omega} p^{\alpha+\beta-1}(t, x) w(x) d x\right)^{2}} \\
& {\left[\operatorname{mes}(\Omega) \int_{\Omega}\left(p^{\alpha+\beta-2}(t, x) w(x)\right)^{2} d x-\left(\int_{\Omega} p^{\alpha+\beta-2}(t, x) w(x) d x\right)^{2}\right]}
\end{aligned}
$$

Applying inequality (2.22) for $f=p^{\alpha+\beta-2} w$ and $g=h$, we get 


$$
\begin{aligned}
& \left(\operatorname{mes}(\Omega) \int_{\Omega} h(x) w(x) p^{\alpha+\beta-2}(t, x) d x-\int_{\Omega} h(x) d x \int_{\Omega} p^{\alpha+\beta-2}(t, x) w(x) d x\right)^{2} \\
& \leq\left(\operatorname{mes}(\Omega) \int_{\Omega}\left(p^{\alpha+\beta-2}(t, x) w(x)\right)^{2} d x-\left(\int_{\Omega} p^{\alpha+\beta-2}(t, x) w(x) d x\right)^{2}\right) \\
& \quad \times\left(\operatorname{mes}(\Omega) \int_{\Omega} h^{2}(x) d x-\left(\int_{\Omega} h(x) d x\right)^{2}\right)
\end{aligned}
$$

Hence, we obtain $\dot{M}(p) \leq 0 . \dot{M}(p)=0$ holds if and only if

$$
\exists \sigma_{t}, \rho_{t} \in \mathbb{R} \quad \text { such that } \quad w p^{\alpha+\beta-2}=\sigma_{t} h+\rho_{t} .
$$

The general form of the equilibrium density is given by

$$
w(x) p^{\alpha+\beta-2}(t, x)=\frac{C_{t} \lambda_{1}^{\prime} h(x)}{\gamma}+\frac{C_{t} \lambda_{2}^{\prime}}{\gamma}
$$

where $C_{t}=(\beta-\alpha) \int_{\Omega} p^{\alpha+\beta-1}(t, x) w(x) d x /(\alpha+\beta-1)$.

For $\alpha+\beta=3$, applying equations (2.3), (4.9) and (4.14), we have $\sigma_{t}=\sigma=$ $\lambda_{1}^{\prime}\left(C_{t} / \gamma\right)$ and $\rho_{t}=\rho=\lambda_{2}\left(C_{t} / \gamma\right)$, having an unique density function $p^{*}(x)$ corresponding to the maximum entropy distribution that satisfies equations (2.3) and (4.9) for an equilibrium state of a non-stationary system having evolution equation given by (4.10).

\section{Asymptotic convergence}

Asymptotic convergence in this case can be obtained similar to the previous case applying Barbalat's lemma and by considering that the weighted function $w$ is bounded.

Theorem 4.2. If $p(t, x)$ is a density function given by equation

$$
u=-\gamma \nabla_{u} \dot{\widetilde{V^{w}}}+\lambda_{1}^{\prime} h+\lambda_{2}^{\prime}
$$

then for all $x$ we have $\lim _{t \rightarrow \infty} p(t, x)=p^{*}(x)$. 


\section{Rényi-Tsallis entropy of order $\alpha$ and non-stationary systems}

Tsallis entropy of a continuous random variable $X_{t}, t \geq 0$ is defined as

$$
S_{\alpha}\left(X_{t}\right)=\frac{1}{\alpha-1}\left(\int_{\Omega} p^{\alpha}(t, x) d x-1\right), \quad \alpha>0, \alpha \neq 1 .
$$

Tsallis entropy has many applications, for example, in image thresholding, information theory and stellar polytropes studies [38, 6, 39].

Rényi-Tsallis entropy of order $\alpha$ in the discrete case was introduced in 2017 by Wondie and Kumar [40]; this entropy is defined as

$$
H^{\alpha}(p)=\frac{1}{\alpha^{-1}-\alpha}\left[\log \left(\sum_{k=1}^{n} p_{k}^{\alpha}\right)+\sum_{k=1}^{n} p_{k}^{\alpha}-1\right], \quad \alpha>0, \alpha \neq 1 .
$$

For $\alpha \rightarrow 1$, we get the Shannon entropy. Rényi-Tsallis entropy of order $\alpha$ has most of the properties of Shannon entropy.

Rényi-Tsallis entropy of order $\alpha$ is symmetric, nonnegative, concave for $0<$ $\alpha<1$ and continuous. In this section, we introduce the Rényi-Tsallis entropy of order $\alpha$ in the continuous case which preserves the same properties as in the discrete case.

Rényi-Tsallis entropy of order $\alpha$ of a random continuous variable $X_{t}, t \geq 0$ is defined as

$$
H^{\alpha}\left(X_{t}\right)=\frac{1}{\alpha^{-1}-\alpha}\left[\log \left(\int_{\Omega} p^{\alpha}(t, x) d x\right)+\int_{\Omega} p^{\alpha}(t, x) d x-1\right], \quad \alpha>0, \alpha \neq 1
$$

For $\alpha \rightarrow 1$, we obtain the Shannon entropy.

\subsection{Speed-gradient and maximum Rényi-Tsallis entropy of order $\alpha$ principles}

Applying the SG principle for non-stationary processes $\left(X_{t}\right)_{t \geq 0}$, we determine the speed of the goal functional $\dot{\widetilde{H^{\alpha}}}=\frac{d \widetilde{H^{\alpha}}}{d t}$ as

$$
\dot{\widetilde{H^{\alpha}}}\left(X_{t}\right)=-\frac{\alpha}{\alpha^{-1}-\alpha}\left(\int_{\Omega} u(t, x) p^{\alpha-1}(t, x) d x\right)\left[\frac{1+\int_{\Omega} p^{\alpha}(t, x) d x}{\int_{\Omega} p^{\alpha}(t, x) d x}\right]
$$


where $\widetilde{H^{\alpha}}=-H^{\alpha}$.

The gradient of $\dot{\hat{H}^{\alpha}}$ by $u$ is equal to

$$
\nabla_{u} \dot{\widetilde{H^{\alpha}}}\left(X_{t}\right)=-\frac{\alpha}{\alpha^{-1}-\alpha} p^{\alpha-1}(t, x)\left(\frac{1+\int_{\Omega} p^{\alpha}(t, x) d x}{\int_{\Omega} p^{\alpha}(t, x) d x}\right)
$$

According to the SG principle, we obtain the following evolution law

$$
u=\frac{\gamma \alpha}{\alpha^{-1}-\alpha} p^{\alpha-1}(t, x)\left(\frac{1+\int_{\Omega} p^{\alpha}(t, x) d x}{\int_{\Omega} p^{\alpha}(t, x) d x}\right)+\lambda^{\prime}
$$

where $\lambda^{\prime}$ is the Lagrange multiplier which obeys the following condition

$$
\int_{\Omega}\left(\frac{\gamma \alpha}{\alpha^{-1}-\alpha} p^{\alpha-1}(t, x)\left(\frac{1+\int_{\Omega} p^{\alpha}(t, x) d x}{\int_{\Omega} p^{\alpha}(t, x) d x}\right)+\lambda^{\prime}\right) d x=0
$$

From this we obtain the Lagrange multiplier as

$$
\lambda^{\prime}=-\frac{\gamma \alpha}{\left(\alpha^{-1}-\alpha\right) \operatorname{mes}(\Omega)}\left(\frac{1+\int_{\Omega} p^{\alpha}(t, x) d x}{\int_{\Omega} p^{\alpha}(t, x) d x}\right) \int_{\Omega} p^{\alpha-1}(t, x) d x
$$

The system dynamics equation has the following form

$$
\dot{p}(t, x)=\frac{\gamma \alpha}{\alpha^{-1}-\alpha}\left(\frac{1+\int_{\Omega} p^{\alpha}(t, x) d x}{\int_{\Omega} p^{\alpha}(t, x) d x}\right)\left[p^{\alpha-1}(t, x)-\frac{\int_{\Omega} p^{\alpha-1}(t, x) d x}{m e s(\Omega)}\right]
$$

The general form of the evolution of the system is

$$
\dot{p}=-\frac{\gamma}{C_{t}}(\Psi-I) p^{\alpha-1}
$$

where $\Psi=\int_{\Omega}(\dot{)} d x / \operatorname{mes}(\Omega)$ is a linear operator which is invariant to $p, I$ is an identity operator and $C_{t}=\frac{\left(\alpha^{-1}-\alpha\right) \int_{\Omega} p^{\alpha}(t, x) d x}{\alpha\left(1+\int_{\Omega} p^{\alpha}(t, x) d x\right)}$.

The stability of the obtained equilibrium (5.9) is derived considering the following Lyapunov function $M(p)=\widetilde{H^{\alpha}}\left(X_{t}\right)-\widetilde{H^{\alpha}}{ }_{\min }\left(X_{t}\right) \geq 0$ where $\widetilde{H^{\alpha}}{ }_{\text {min }}\left(X_{t}\right)$ is the minimum of $\widetilde{H^{\alpha}}\left(X_{t}\right)$. We have the following 


$$
\begin{aligned}
\dot{M}(p) & =\frac{\gamma \alpha^{2}}{\left(\alpha^{-1}-\alpha\right)^{2} \operatorname{mes}(\Omega)}\left(\frac{1+\int_{\Omega} p^{\alpha}(t, x) d x}{\int_{\Omega} p^{\alpha}(t, x) d x}\right)^{2} \\
& \times\left[\left(\int_{\Omega} p^{\alpha-1}(t, x) d x\right)^{2}-\operatorname{mes}(\Omega) \int_{\Omega}\left(p^{\alpha-1}(t, x) d x\right)^{2} d x\right]
\end{aligned}
$$

Applying the CBS inequality (2.14) for $f=p^{\alpha-1}$ and $g=1$, we obtain $\dot{M}(p) \leq 0$ is true when $p^{\alpha-1}(t, x)=\sigma_{t}$ which is possible only if we take $p(t, x)=C=$ constant. Applying equation (2.3), we prove that $\sigma_{t}$ does not depend on $t$ and we derive $C$ to be $C=(\text { mes }(\Omega))^{-1}$. Therefore, there is only one unique density function $p^{*}=(\text { mes }(\Omega))^{-1}$ corresponding to the maximum entropy distribution that satisfies equation (2.3) for the equilibrium state of the system which evolves by evolution law given by (5.9).

\section{Asymptotic convergence}

Applying Barbalat's lemma the asymptotic convergence of all solutions to $p^{*}$ can be proven.

Theorem 5.1. If $p(t, x)$ is a density function defined by equation (5.9), then for any $x$ we get $\lim _{t \rightarrow \infty} p(t, x)=p^{*}(x)$.

\subsection{Total energy constraint}

Consider a system having a total energy constraint given by [27]

$$
\int_{\Omega} p(t, x) h(x) d x=E,
$$

where $E$ is the common energy of a system and $h$ is the density of energy, $h \in L_{2}(\Omega), h$ is not a constant function.

The dynamics equation of this system is defined as [27]

$$
u=-\gamma \nabla_{u} \dot{H}^{\alpha}+\lambda_{1} h+\lambda_{2}
$$

We have

$$
u=-\frac{\gamma \alpha}{\alpha^{-1}-\alpha} p^{\alpha-1}(t, x)\left(\frac{1+\int_{\Omega} p^{\alpha}(t, x) d x}{\int_{\Omega} p^{\alpha}(t, x) d x}\right)+\lambda_{1} h(x)+\lambda_{2}
$$

Based on constraints (2.3) and (5.12), the Lagrange multipliers $\lambda_{1}$ and $\lambda_{2}$ are 


$$
\begin{aligned}
\lambda_{1} & =\frac{\gamma \alpha}{\alpha^{-1}-\alpha}\left(\frac{1+\int_{\Omega} p^{\alpha}(t, x) d x}{\int_{\Omega} p^{\alpha}(t, x)}\right) \\
& \times \frac{m e s(\Omega) \int_{\Omega} h(x) p^{\alpha-1}(t, x) d x-\int_{\Omega} h(x) d x \int_{\Omega} p^{\alpha-1}(t, x) d x}{\operatorname{mes}(\Omega) \int_{\Omega} h^{2}(x) d x-\left(\int_{\Omega} h(x) d x\right)^{2}} \\
\lambda_{2}= & \frac{\gamma \alpha}{\alpha^{-1}-\alpha}\left(\frac{1+\int_{\Omega} p^{\alpha}(t, x) d x}{\int_{\Omega} p^{\alpha}(t, x)}\right) \\
\times & \frac{\int_{\Omega} h^{2}(x) d x \int_{\Omega} p^{\alpha-1}(t, x) d x-\int_{\Omega} h(x) p^{\alpha-1}(t, x) d x \int_{\Omega} h(x) d x}{m e s(\Omega) \int_{\Omega} h^{2}(x) d x-\left(\int_{\Omega} h(x) d x\right)^{2}}
\end{aligned}
$$

\section{Equilibrium stability}

Using the same Lyapunov function $M$ used in the previous section for $\widetilde{H^{\alpha}}$ we obtain the corresponding Lagrange multipliers $\lambda_{1}^{\prime}=-\lambda_{1}, \lambda_{2}^{\prime}=-\lambda_{2}$ and the following expression

$$
\begin{aligned}
& \dot{M}(p)=\frac{\gamma \alpha^{2}}{\left(\alpha^{-1}-\alpha\right)^{2}}\left(\frac{1+\int_{\Omega} p^{\alpha}(t, x) d x}{\int_{\Omega} p^{\alpha}(t, x) d x}\right)^{2} \frac{1}{\operatorname{mes}(\Omega) \int_{\Omega} h^{2}(x) d x-\left(\int_{\Omega} h(x) d x\right)^{2}} \\
& \times\left[-\operatorname{mes}(\Omega) \int_{\Omega}\left(p^{\alpha-1}(t, x)\right)^{2} d x \int_{\Omega} h^{2}(x) d x+\int_{\Omega}\left(p^{\alpha-1}(t, x)\right)^{2} d x\left(\int_{\Omega} h(x) d x\right)^{2}\right. \\
& +\operatorname{mes}(\Omega)\left(\int_{\Omega} h(x) p^{\alpha-1}(t, x) d x\right)^{2}-2 \int_{\Omega} h(x) p^{\alpha-1}(t, x) d x \\
& \left.\times \int_{\Omega} h(x) d x \int_{\Omega} p^{\alpha-1}(t, x) d x+\left(\int_{\Omega} p^{\alpha-1}(t, x) d x\right)^{2} \int_{\Omega} h^{2}(x) d x\right]
\end{aligned}
$$

Applying inequality (2.22) for $f=p^{\alpha-1}$ and $g=h$, we get 


$$
\begin{aligned}
& \left(\operatorname{mes}(\Omega) \int_{\Omega} h(x) p^{\alpha-1}(t, x) d x-\int_{\Omega} h(x) d x \int_{\Omega} p^{\alpha-1}(t, x) d x\right)^{2} \\
& \leq\left(\operatorname{mes}(\Omega) \int_{\Omega}\left(p^{\alpha-1}(t, x)\right)^{2} d x-\left(\int_{\Omega} p^{\alpha-1}(t, x) d x\right)^{2}\right) \\
& \quad \times\left(\operatorname{mes}(\Omega) \int_{\Omega} h^{2}(x) d x-\left(\int_{\Omega} h(x) d x\right)^{2}\right)
\end{aligned}
$$

Therefore, we obtain $\dot{M}(p) \leq 0 . \dot{M}=0$ holds if and only if

$$
\exists \quad \sigma_{t}, \rho_{t} \in \mathbb{R} \quad \text { such that } p^{\alpha-1}=\sigma_{t} h+\rho_{t} .
$$

The density equilibrium is given by

$$
p^{\alpha-1}(t, x)=\frac{C_{t} \lambda_{1}^{\prime} h(x)}{\gamma}+\frac{C_{t} \lambda_{2}^{\prime}}{\gamma}
$$

where $C_{t}=\frac{\left(\alpha-\alpha^{-1}\right) \int_{\Omega} p^{\alpha}(t, x) d x}{\alpha\left(1+\int_{\Omega} p^{\alpha}(t, x) d x\right)}$.

For $\alpha=2$, applying equations (2.3), (5.12) and 5.17, we have that $\sigma_{t}$ and $\rho_{t}$ do not depend on $t$. Hence, we have $\sigma_{t}=\sigma=\lambda_{1}^{\prime}\left(C_{t} / \gamma\right)$ and $\rho_{t}=\rho=\lambda_{2}^{\prime}\left(C_{t} / \gamma\right)$. In this case, we have an unique distribution for the equilibrium state $p^{*}(x)$.

\section{Asymptotic convergence}

Similar as in the previous sections, the asymptotic convergence is obtained.

Theorem 5.2. If $p(t, x)$ is a density function defined by equation

$$
u=-\gamma \nabla_{u} \dot{\tilde{H}^{\alpha}}+\lambda_{1}^{\prime} h+\lambda_{2}^{\prime},
$$

then for any $x$ we get $\lim _{t \rightarrow \infty} p(t, x)=p^{*}(x)$.

\section{Conclusion}

Evolution of a physical system over time has long been studied over the decades being an important topic in the specialized literature. It is desired 
for systems to tend to only one state while this state should be of equilibrium and the system should converge to it.

This paper studied the dynamics of non-stationary processes in the context of maximum Varma entropy principle, maximum weighted Rényi principle, maximum weighted Varma entropy and maximum Rényi-Tsallis entropy of order $\alpha$ principles and derived the corresponding unique density function for the equilibrium state of the system. We have obtained equations (2.10), (3.8), (4.7) and (5.9) which describe the dynamics of non-stationary systems; systems that as time passes tend to the maximum entropy state.

We introduced the weighted Varma entropy and Rényi-Tsallis entropy of order $\alpha$ in the continuous case. Varma entropy is a generalization of Rényi entropy having two parameters. We believe, because of the two parameters, Varma entropy is more flexible than Rényi entropy. Weighted entropies are designed to give different importance to data, being more complex. RényiTsallis entropy of order $\alpha$ in the continuous case has the same properties as in the discrete case being more complex and flexible for applications.

\section{References}

[1] T. Cover, J. A. Thomas, Elements of Information Theory, second ed., John Wiley \& Sons Inc., Hoboken, NJ, 2006.

[2] C. Bălcău, C. Niculescu, Linearly constrained Iosifescu-Theodorescu entropy maximization for homogeneous stationary multiple Markov chains, Mathematical Reports 14 (3) (2012), 243-251.

[3] C. Bălcău, Maximum Entropy Methods for Countable Markov Chains, Analele Universităţii Bucureşti 131 (2003).

[4] M. P. De Albuquerque, I. A. Esquef, A. G. Mello, Image thresholding using Tsallis entropy, Pattern Recognition Letters 25 (9) (2004), 1059-1065.

[5] A. R. Plastino, A. Plastino, Tsallis' entropy, Ehrenfest theorem and information theory, Physics Letters A 177 (3) (1993), 177-179.

[6] A. K. Bhandari, A. Kumar, G. K. Singh, Tsallis entropy based multilevel thresholding for colored satellite image segmentation using evolutionary algorithms, Expert Systems with Applications 42 (22) (2015), 8707-8730. 
[7] G. Kaniadakis, Relativistic entropy and related Boltzmann kinetics, The European Physical Journal A 40 (3) (2009), 275.

[8] K. Ourabah, A. H. Hamici-Bendimerad, M. Tribeche, Quantum entanglement and Kaniadakis entropy, Physica Scripta 90 (4) (2015), 045101 .

[9] A. Y. Abul-Magd, Nonextensive random-matrix theory based on Kaniadakis entropy, Physics Letters A 361(6) (2007), 450-454.

[10] V. Kumar, H. C. Taneja, Some characterization results on generalized cumulative residual entropy measure, Statistics \& Probability Letters 81 (8) (2011), 1072-1077.

[11] R. Kleeman, Measuring dynamical prediction utility using relative entropy, Journal of the atmospheric sciences 59 (13) (2002), 2057-2072.

[12] H. Liu, W. Chen, A. Sudjianto, Relative entropy based method for probabilistic sensitivity analysis in engineering design, Journal of Mechanical Design 128 (2) (2006), 326-336.

[13] S. Furuichi, K. Yanagi, K. Kuriyama, Fundamental properties of Tsallis relative entropy, Journal of Mathematical Physics 45 (12) (2004), 4868-4877.

[14] M. Belis, S. Guiasu, A quantitative-qualitative measure of information in cybernetic systems (Corresp.), IEEE Transactions on Information Theory 14 (4) (1968), 593-594.

[15] S. Guiasu, Weighted entropy, Reports on Mathematical Physics 2 (3) (1971), 165-179.

[16] G. Dial, I. J. Taneja, On weighted entropy of type $(\alpha, \beta)$ and its generalizations, Aplikace matematiky 26 (6) (1981), 418-425.

[17] J. Bruhn, L. E. Lehmann, H. Rpcke, T. W. Bouillon, A. Hoeft, Shannon entropy applied to the measurement of the electroencephalographic effects of desflurane, Anesthesiology: The Journal of the American Society of Anesthesiologists 95 (1) (2001), 30-35.

[18] D. E. Lake, Rényi entropy measures of heart rate Gaussianity, IEEE Transactions on Biomedical Engineering 53 (1) (2006), 21-27. 
[19] V. S. Barbu, A. Karagrigoriou, V. Preda, Entropy and divergence rates for Markov chains: I. The Alpha-Gamma and Beta-Gamma case, Proceedings of the Romanian Academy-series A 4 (2017).

[20] V. S. Barbu, A. Karagrigoriou, V. Preda, Entropy and divergence rates for Markov chains: II. The weighted case, Proceedings of the Romanian Academy-series A 1 (2018).

[21] V. S. Barbu, A. Karagrigoriou, V. Preda, Entropy and divergence rates for Markov chains: III. The Cressie and Read case and applications, Proceedings of the Romanian Academy-series A 2 (2018).

[22] R. S. Varma, Generalization of Rényi's entropy of order $\alpha$, Journal of Mathematical Sciences 1 (1966), 34-48.

[23] R. Thapliyal, H. C. Taneja, Generalized entropy of order statistics, Applied Mathematics 3 (12) (2012), 1977.

[24] S. Kayal, Some results on dynamic discrimination measures of order $(\alpha, \beta)$, Hacettepe Journal of Mathematics and Statistics 44 (1) (2015), 179-188.

[25] J. N. Kapur, H. K. Kesavan, Entropy optimization principles and their applications, in: Entropy and energy dissipation in water resources, Springer, Dordrecht, 1992, 3-20.

[26] V. Preda, S. Dedu, C. Gheorghe, New classes of Lorenz curves by maximizing Tsallis entropy under mean and Gini equality and inequality constraints, Physica A: Statistical Mechanics and its Applications 436 (2015), 925-932.

[27] D. S. Shalymov, A. L. Fradkov, Dynamics of non-stationary processes that follow the maximum of the Rényi entropy principle, Proceedings of the Royal Society A: Mathematical, Physical and Engineering Sciences 472 (2185) (2016), 20150324.

[28] D. Shalymov, Dynamics of non-stationary processes that follow the maximum of continuous Tsallis entropy, Cybernetics Phys 5 (2016), 5966.

[29] A. L. Fradkov, Speed-gradient scheme and its application in adaptive control problems, Automation and Remote Control 40 (9) (1980), 13331342 . 
[30] A. Fradkov, Speed-gradient entropy principle for nonstationary processes, Entropy 10 (4) (2008), 757-764.

[31] P. A. Bromiley, N. A. Thacker, E. Bouhova-Thacker, Shannon entropy, Rényi entropy, and information, Statistics and Inf. Series (2004).

[32] A. Stuart, J. K. Ord, Kendall's Advanced Theory of Statistics: Distribution theory; Vol. 2, Classical inference and relationship; Vol. 3, Design and analysis, and time-series, Charles Griffin, 1987.

[33] M. Hermanns, S. Trebst, Rényi entropies for classical string-net models, Physical Review B 89 (20) (2014), 205107.

[34] G. Chicco, J. S. Akilimali, Rényi entropy-based classification of daily electrical load patterns, IET generation, transmission \& distribution $\mathbf{4}$ (6) (2010), 736-745.

[35] D. Aiordachioaie, Signal segmentation based on direct use of statistical moments and Rényi entropy, in: 2013 International Conference on Electronics, Computer and Computation (ICECCO), IEEE, 2013, 359362 .

[36] J. Dayou, N. C. Han, H. C. Mun, A. H. Ahmad, S. V. Muniandy, M. N. Dalimin, Classification and identification of frog sound based on entropy approach, in: 2011 International Conference on Life Science and Technology 3 (2011), 184-187.

[37] A. M. Lyapunov, Stability of motion, New York, NY: Academic Press, 1966.

[38] M. P. De Albuquerque, I. A. Esquef, A. G. Mello, Image thresholding using Tsallis entropy, Pattern Recognition Letters 25 (9) (2004), 1059-1065.

[39] A. R. Plastino, A. Plastino, Stellar polytropes and Tsallis' entropy, Physics Letters A 174 (5-6) (1993), 384-386.

[40] L. Wondie, S. Kumar, A joint representation of Rényis and Tsalli's entropy with application in coding theory, International Journal of Mathematics and Mathematical Sciences (2017). 
Vasile Preda

"Gheorghe Mihoc-Caius Iacob" Institute of Mathematical Statistics and Applied Mathematics, Bucharest, Romania

"Costin C. Kiriţescu" National Institute of Economic Research, Bucharest, Romania

Faculty of Mathematics and Computer Science, University of Bucharest, Romania E-mail: vasilepreda0@gmail.com

Irina Băncescu

"Costin C. Kiriţescu" National Institute of Economic Research, Bucharest, Romania

E-mail: irina_adrianna@yahoo.com 